\title{
Multiple-trait genomic evaluation of linear type traits using genomic and phenotypic data in US Holsteins
}

\author{
S. Tsuruta, ${ }^{* 1}$ I. Misztal, ${ }^{*}$ I. Aguilar, $†$ and T. J. Lawlorł \\ *Animal and Dairy Science Department, University of Georgia, Athens 30602 \\ †Instituto Nacional de Investigación Agropecuaria, Las Piedras, Canelones 90200, Uruguay \\ ¥Holstein Association USA Inc., Brattleboro, VT 05301
}

\section{ABSTRACT}

Currently, the USDA uses a single-trait (ST) model with several intermediate steps to obtain genomic evaluations for US Holsteins. In this study, genomic evaluations for 18 linear type traits were obtained with a multiple-trait (MT) model using a unified singlestep procedure. The phenotypic type data on up to 18 traits were available for 4,813,726 Holsteins, and single nucleotide polymorphism markers from the Illumina BovineSNP50 genotyping Beadchip (Illumina Inc., San Diego, CA) were available on 17,293 bulls. Genomic predictions were computed with several genomic relationship matrices $(\mathbf{G})$ that assumed different allele frequencies: equal, base, current, and current scaled. Computations were carried out with ST and MT models. Procedures were compared by coefficients of determination $\left(\mathrm{R}^{2}\right)$ and regression of 2004 prediction of bulls with no daughters in 2004 on daughter deviations of those bulls in 2009. Predictions for 2004 also included parent averages without the use of genomic information. The $\mathrm{R}^{2}$ for parent averages ranged from 10 to $34 \%$ for ST models and from 12 to $35 \%$ for MT models. The average $\mathrm{R}^{2}$ for all $\mathbf{G}$ were 34 and $37 \%$ for ST and MT models, respectively. All of the regression coefficients were $<1.0$, indicating that estimated breeding values in 2009 of 1,307 genotyped young bulls' parents tended to be biased. The average regression coefficients ranged from 0.74 to 0.79 and from 0.75 to 0.80 for ST and MT models, respectively. When the weight for the inverse of the numerator relationship matrix $\left(\mathbf{A}^{-1}\right)$ for genotyped animals was reduced from 1 to $0.7, \mathrm{R}^{2}$ remained almost identical while the regression coefficients increased by $0.11-0.26$ and $0.12-0.23$ for ST and MT models, respectively. The ST models required about $5 \mathrm{~s}$ per iteration, whereas MT models required 3 (6) min per iteration for the regular (genomic) model. The MT single-step approach is feasible for 18 linear

Received February 9, 2011.

Accepted April 8, 2011.

${ }^{1}$ Corresponding author: shogo@uga.edu type traits in US Holstein cattle. Accuracy for genomic evaluation increases when switching ST models to MT models. Inflation of genomic evaluations for young bulls could be reduced by choosing a small weight for the $\mathbf{A}^{-1}$ for genotyped bulls.

Key words: genomic evaluation, linear type trait, US Holstein

\section{INTRODUCTION}

Genomic selection in US Holsteins has been conducted by the USDA-ARS Animal Improvement Programs Laboratory (Beltsville, MD) since 2008, using a multi-step procedure (MSP), where regular PTA are used to create genomic predictions (VanRaden, 2008). The MSP uses a single-trait (ST) model, and improved genomic relationships influence only the genotyped animals.

Misztal et al. (2009) proposed that genomic evaluations be performed in a single-step procedure (SSP) with complete phenotypic, pedigree, and genomic information. Aguilar et al. (2010) applied the SSP to obtain genomic EBV (GEBV) for final score of US Holsteins, where a pedigree-based relationship matrix $(\mathbf{A})$ in the evaluation procedure is replaced by a matrix $(\mathbf{H})$ that combines $\mathbf{A}$ and a genomic relationship matrix $(\mathbf{G})$. They reported that accuracy and inflation of genomic evaluation varied with different $\mathbf{G}$, with the best $\mathbf{G}$ derived using equal allele frequencies $(\mathbf{G E})$. The inflation of genomic evaluation could be reduced or eliminated with small modifications to the $\mathbf{H}$ matrix. Computing time of SSP with the ST model was close to that of a regular BLUP procedure with $\mathbf{A}$.

Performance of SSP has been evaluated in other species. Chen et al. (2011) used SSP to analyze 3 traits in 2 separately selected lines of chickens. The improvement in accuracy after adding the genomic information varied between the 2 lines, despite similar heritability for 3 traits. Variation in performance of the SSP in the 2 lines was attributed to the different selection goals. Forni et al. (2011) used SSP to analyze litter sizes in pigs. Predictions obtained with different $\mathbf{G}$ were highly 
correlated; however, heritability estimates varied by type of $\mathbf{G}$, whereas heritability estimates were the same as with $\mathbf{G}$ scaled similarly to $\mathbf{A}$.

Chen et al. (2011) investigated the effect of using different $\mathbf{G}$ and their scaling in chickens. Accuracy with $\mathbf{G}$ assuming current allele frequencies (GC) was marginally higher than that assuming GE. However, predictions from both $\mathbf{G}$ were biased. The bias could be eliminated by shifting $\mathbf{G}$ so that averages of offdiagonals in $\mathbf{G}$ and in $\mathbf{A}$ for genotyped animals were equal within 0.001 . The bias due to the incorrect offset of $\mathbf{G}$ was greater when the training population had low accuracy, and increased with stronger selection (Vitezica et al., 2010).

A multiple-trait (MT) animal model is currently used for the regular national genetic evaluation of linear type traits in US Holsteins. As more selection decisions are being made utilizing genomic information, it becomes critical that all genomic information be included in a single national evaluation. Substantial bias in genomic evaluations caused by selection on genotyped animals was reported by simulation studies of Patry and Ducrocq (2009) and Liu et al. (2009). The objectives of this study were to evaluate the feasibility of an MT SSP using a large number of animals for 18 linear type traits in Holsteins for routine use, quantify the improvement in accuracy using an MT SSP compared with an ST SSP, and investigate how modifications to $\mathbf{H}$, which combines $\mathbf{G}$ and $\mathbf{A}$, reduce the inflation of GEBV.

\section{MATERIALS AND METHODS}

\section{Data}

Genetic SNP markers from the Illumina BovineSNP50 genotyping Beadchip (Illumina Inc., San Diego, CA), consisting of 38,416 informative SNP, were available on 17,293 Holstein bulls. Those SNP markers were converted to 0,1 , or 2 for calculation of $\mathbf{G}$ as described in Aguilar et al. (2010). Two data sets, a reduced file having data up to the year 2004 and a full file having data up to the year 2009, were used. Comparing early predictions from the reduced 2004 file with the more recent 2009 data allowed us to assess reductions in bias and accuracy. Pedigree information included 7,860,183 animals for cows with phenotypes and bulls with genotypes. The 2004 data set included 7,715,925 phenotypic records on 4,813,726 cows. The 2009 data set included $8,865,120$ phenotypic records of $5,657,787$ cows. Of the 17,293 genotyped bulls, 6,913 bulls had genotypes in 2004 . Of the 6,913 bulls, 1,307 had no daughters with phenotypes in 2004 and at least 50 daughters with phenotypes in 2009 .

\section{Model}

The MT animal model used in the genetic evaluation for 18 linear type traits is described in Tsuruta et al. (2005). In the genomic evaluation, the inverse of $\mathbf{H}$ $\left(\mathbf{H}^{-1}\right)$ replaced the inverse of $\mathbf{A}\left(\mathbf{A}^{-1}\right)$. Aguilar et al. (2010) and Christensen and Lund (2010) describe the decomposition of $\mathbf{H}^{-1}$ as follows:

$$
\mathbf{H}^{-1}=\left[\begin{array}{cc}
\mathbf{A}^{11} & \mathbf{A}^{12} \\
\mathbf{A}^{21} & \mathbf{A}^{22}+\tau \mathbf{G}^{-1}-\omega \mathbf{A}_{22}^{-1}
\end{array}\right],
$$

where $\mathbf{A}_{22}$ is a pedigree-based numerator relationship matrix for 6,913 genotyped animals, and $\tau$ and $\omega$ are weight factors for $\mathbf{G}^{-1}$ and $\mathbf{A}_{22}^{-1}$, respectively. Using phenotypes and $\mathbf{A}^{-1}, \mathrm{EBV}$ in 2004 and 2009 were calculated with traditional ST and MT models. Using phenotypes and $\mathbf{H}^{-1}$ with pedigree and genomic relationships, GEBV in 2004 were calculated with ST SSP and MT SSP, varying the weight $(\omega)$ on $\mathbf{A}_{22}^{-1}$ for genotyped animals. Changes in weights of $\mathbf{G}^{-1}$ were not investigated $(\tau=1)$ as this parameter had a small effect on accuracy and inflation (Misztal et al., 2010). The G used in our SSP genomic evaluations were centered on 4 different allele frequencies: $p=q=0.5$ (GE), base population allele frequencies calculated with the method of Gengler et al. (2007) (GB), derived directly from the genomic information (GC), and current allele frequencies with $\mathbf{G}$ scaled to match A (GCS; Chen et al., 2011).

\section{Computation}

To assess the bias of GEBV, regression coefficients ( $\boldsymbol{\delta})$ of daughter deviations (DD), defined by VanRaden (2008), for traditional EBV in 2009 (EBV09) on GEBV in 2004 were calculated for the 1,307 young bulls with no daughters in the 2004 and with at least 50 daughters in 2009. Coefficients of determination $\left(\mathrm{R}^{2}\right)$ of the regression models were also calculated for the same bulls to quantify the accuracy of GEBV. Traditional EBV in 2004 were used to calculate parent average (PA). All EBV and GEBV were computed with the modified BLUP90IOD program (Tsuruta et al., 2001), which uses iteration on data with preconditioned conjugate gradient, on an Intel Xeon server with 2.93-GHz clock speed and $12 \mathrm{MB}$ of cache memory. To improve the convergence rate, the preconditioned conjugate gradient algorithm used a block preconditioner (size $=$ 18) for MT models (Tsuruta and Misztal, 2008). The squared ratio of the norms of residual and right-handside vectors for $\mathbf{C x}=\mathbf{b}$ (where $\mathbf{C}=$ coefficient matrix, 
$\mathbf{x}=$ solution vector, and $\mathbf{b}=$ observation vector in a system of equations) was used as an indication of iterative convergence (i.e., $\|\mathbf{b}-\mathbf{C x}\|^{2} /\|\mathbf{b}\|^{2}$ ). Convergence criteria ranging from $10^{-11}$ to $10^{-16}$ and from $10^{-11}$ to $10^{-12}$ were used to stop the program for ST and MT models, respectively.

\section{RESULTS AND DISCUSSION}

Almost all of the older genotyped bulls (born before 2000) had classified daughters (Figure 1). The genomic evaluations of genotyped young bulls born after 2004 had limited to no daughter information. Thus, the group of bulls providing the basis of comparison for the performance of different models was born between 2001 and 2004. Some of these bulls would have been genotyped, evaluated, and then culled. Slightly fewer than half of the genotyped bulls in this period (2,619 bulls) had no daughters with phenotypic records in 2004, and of 2,619 bulls, 1,307 had at least 50 daughters with records in 2009 as mentioned before.

To assess the convergence rate, the $\mathrm{R}^{2}(\%)$ and the number of rounds to convergence under several convergence criteria are shown for strength as an example of 18 traits in Table 1. Among the ST models, satisfactory convergence was achieved at the criterion of $10^{-16}$. At the $10^{-14}, 10^{-15}$, and $10^{-16}$ levels, differences in $\mathrm{R}^{2}$ with different $\mathbf{G}$ were negligible. At a less conservative criterion of $10^{-11}$, the $\mathrm{R}^{2}$ were 7 to $10 \%$ lower than those at $10^{-14}$, and $\mathrm{R}^{2}$ seemed to be the highest with GE. No improvement was obtained using stricter con- vergence criteria. Therefore, differences in GEBV with different $\mathbf{G}$ in dairy traits; for example, as in Aguilar et al. (2010), may be due to insufficient convergence. The necessary value of the convergence criterion can be determined based upon examination of $\mathrm{R}^{2}$ for PA, as patterns of convergence were similar for PA with different $\mathbf{G}$, with a slight exception for GC. All ST models required about $5 \mathrm{~s}$ per one round of iteration. The number of rounds varied from about 100 with the criterion of $10^{-11}$ to a range of about 240 to 700 with $10^{-14}$

When using a block preconditioner of the preconditioned conjugate gradient algorithm for MT models, the convergence pattern varied significantly. On the other hand, when using a scalar preconditioner, the convergence pattern was smooth, although a large number of iterations ( $>5$ times more than those for MT models, depending on allele frequencies and convergence criteria) was required to reach convergence. Therefore, a scalar preconditioner was not used for MT models. For MT models with the convergence criterion $10^{-11}$, differences in $R^{2}$ with different $\mathbf{G}$ were smaller than for ST models. For the MT model with GB, reducing the criterion to $10^{-12}$ from $10^{-11}$ changed $\mathrm{R}^{2}$ very slightly, with no change for PA. In this study, the criterion of $10^{-11}$ seemed to be satisfactory for MT with GB. The number of rounds at $10^{-11}$ was about 330 with any allele frequencies but increased to $>2,000$ at $10^{-12}$ for all MT models except with GB; the computer time per iteration was 3 min for PA (traditional models) and 6 min with $\mathbf{G}$. To improve the convergence rate for MT

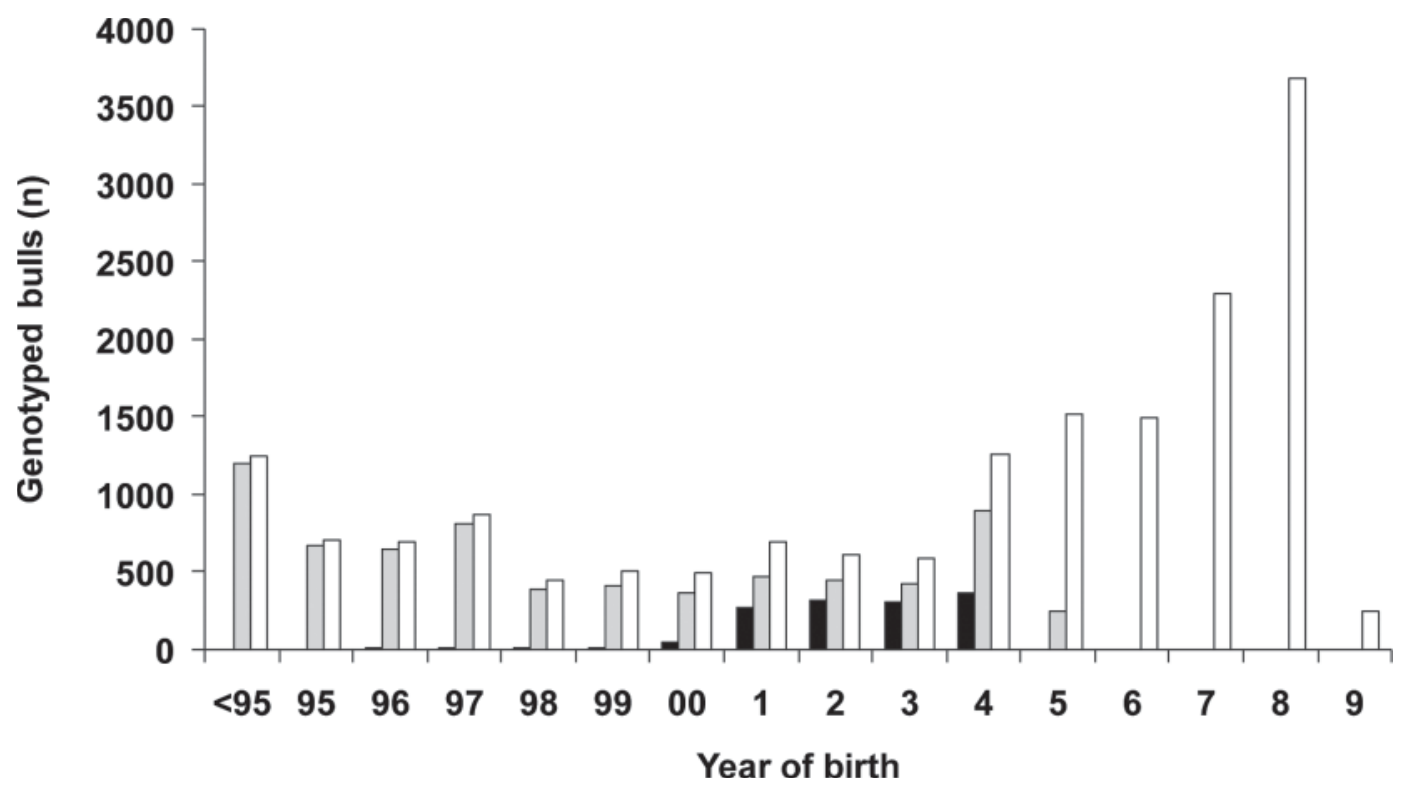

Figure 1. Distributions of genotyped bulls by year of birth: black bars = genotyped bulls with no daughter in 2004 and $>50$ daughters in 2009; gray bars = genotyped bulls with daughters in 2009; white bars = all genotyped bulls. 
Table 1. Coefficients of determination $\left(\mathrm{R}^{2}, \%\right)$ of daughter deviations in 2009 on genomic evaluations of 1,307 genotyped young bulls in 2004 for strength and number of rounds for single-trait and multiple-trait models with equal, base, current, and scaled current allele frequencies (number of rounds in parentheses)

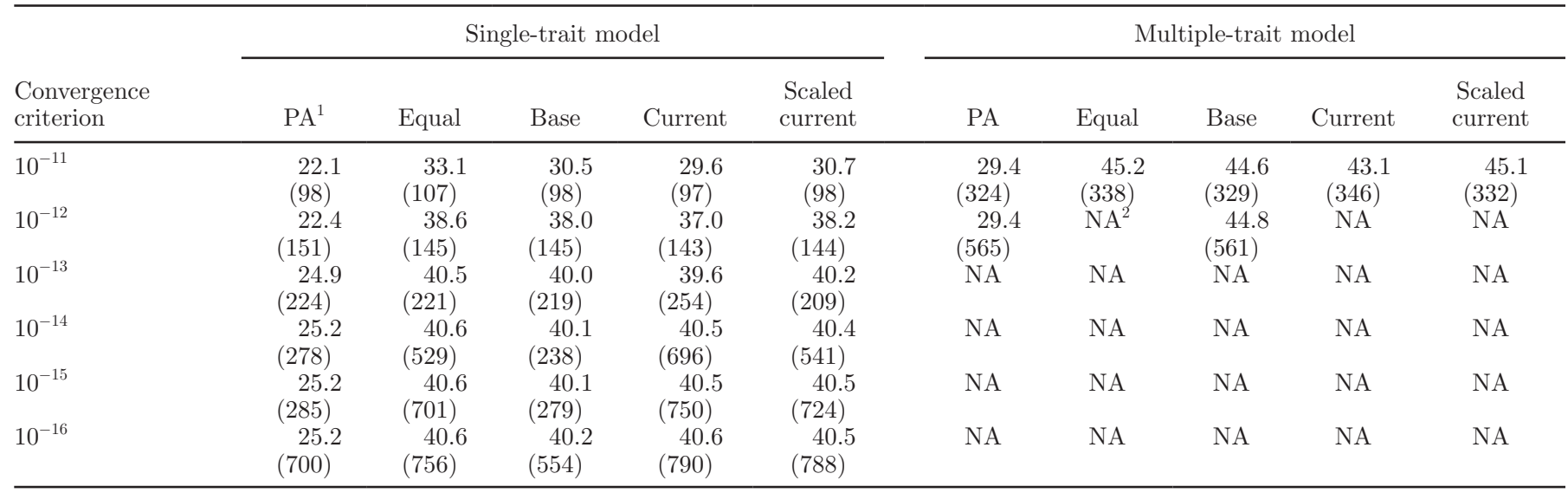

${ }^{1}$ Parent average from genetic evaluation in 2004 .

${ }^{2} \mathrm{NA}=$ not available.

models, the block preconditioner could be modified. Further study is needed to clarify the cause of the slow convergence and the possibility of converging the MT models with other allele frequencies.

The $\mathrm{R}^{2}$ of $\mathrm{DD}$ regressions on GEBV for 1,307 genotyped bulls with no weight for $\mathbf{A}_{22}^{-1}$ are shown in Table 2 . The $\mathrm{R}^{2}$ for PA ranged from 10 to $34 \%$ for ST models and from 12 to $35 \%$ for MT models, with convergence criteria of $10^{-14}$ and $10^{-11}$, respectively. The relatively lower $\mathrm{R}^{2}$ obtained for the last 4 traits (teat length, rear legs rear view, feet and legs score, and rear teat placement) may be due to the recent start of recording for those traits (i.e., $71,69,43$, and $6 \%$ of $7,715,925$ records in the complete file, respectively). The $\mathrm{R}^{2}$ for GEBV were about $16 \%$ higher on average than those for PA. For ST models, $\mathrm{R}^{2}$ for GEBV were similar and averaged $34.6 \%$ with GE, $34.1 \%$ with GB, $34.2 \%$ with GC, and $34.6 \%$ with GCS. Aguilar et al. (2010) reported 4\% difference in $\mathrm{R}^{2}$ among different $\mathbf{G}$ for final score. However, by using a stricter convergence criterion (e.g., $<10^{-13}$ ), differences among those $\mathrm{R}^{2}$ became negligible. Vitezica et al. (2010) and Chen et al. (2011) suggested using GCS as a general way to increase accuracies of GEBV. In those studies, GEBV of the training population had low accuracy, and therefore, incorrectly scaled $\mathbf{G}$ could reduce accuracy for the training population. In dairy, GEBV of the training population had relatively high accuracies and the original $\mathbf{G}$ was similar to $\mathbf{A}$. As a result, the type of $\mathbf{G}$ had a small effect on GEBV of the training population. In general, the improvement of GEBV over EBV is dependent upon the trait and the population structure.

Ideally, $\delta$ values of DD from 2009 on PA and GEBV from 2004 should be 1.0. Table 3 shows the regression coefficients for 1,307 genotyped bulls with at least 50 daughters when no weight for $\mathbf{A}_{22}^{-1}$ (i.e., $\omega=1.0$ ) was used for ST and MT models with the same convergence criteria as in Table 3 (i.e., $10^{-14}$ and $10^{-11}$, respectively). A $\delta$ value $<1.0$ denotes a higher GEBV than DD of EBV09, indicating that GEBV were overestimated or that EBV09 were underestimated. All of the $\delta$ values for PA were <1.0, indicating that EBV09 of 1,307 genotyped young bulls' parents tended to be biased. The average $\delta$ ranged from 74 to 79 and from 75 to 80 for ST and MT models, respectively. In general, the $\delta$ values were similar for all models and traits. For ST and MT models with $\omega=1.0$, the highest values of $\delta$ were obtained when using GE and the lowest with GC. Small differences between $\delta$ values using ST and MT models suggest that biases are not due to selection on correlated type traits. Similar $\delta$ values for all 4 options indicate that biases could be systematic in US Holsteins when using pedigree and genomic information without phenotypic data. Whereas some of the biases can be due to preferential treatment, some of the bias can be due to the structure of a dairy population that cannot be fully modeled. For example, such biases are present in the Israeli population where only young sires are used and there is no preferential treatment $(\mathrm{J}$. Weller, Institute of Animal Science, Bet Dagan, Israel, personal communication, 2010). On average, the bias was smallest and $\mathrm{R}^{2}$ highest with GE. Although the use of GE in broilers resulted in biases (Chen et al., 2011), in dairy the bias due to GE may be partially compensating the bias present in PA, which is systemic to the dairy population.

In a preliminary analysis, we tested various combinations of $\tau$ and $\omega$ ranging from 0.1 to 1.5 and found that 
Table 2. Coefficients of determination $\left(\mathrm{R}^{2}, \%\right)$ of daughter deviations in 2009 on genomic evaluations in 2004 using single-trait and multipletrait models with equal, base, current, and scaled current allele frequencies for genotyped bulls with at least 50 daughters

\begin{tabular}{|c|c|c|c|c|c|c|c|c|c|c|}
\hline Trait & \multicolumn{5}{|c|}{ Single-trait model } & \multicolumn{5}{|c|}{ Multiple-trait model } \\
\hline Strength & 25 & 41 & 40 & 41 & 41 & 29 & 45 & 45 & 43 & 45 \\
\hline Body depth & 28 & 45 & 45 & 45 & 45 & 30 & 48 & 47 & 46 & 48 \\
\hline Dairy form & 20 & 40 & 40 & 39 & 40 & 21 & 42 & 41 & 39 & 41 \\
\hline Rear legs side view & 14 & 27 & 26 & 26 & 27 & 16 & 29 & 29 & 29 & 29 \\
\hline Foot angle & 15 & 25 & 24 & 25 & 25 & 21 & 33 & 32 & 32 & 33 \\
\hline Fore udder attachment & 14 & 34 & 33 & 34 & 34 & 17 & 40 & 39 & 36 & 40 \\
\hline Rear udder height & 18 & 33 & 32 & 33 & 33 & 19 & 36 & 35 & 31 & 36 \\
\hline Rear udder width & 16 & 30 & 29 & 29 & 30 & 18 & 33 & 33 & 29 & 33 \\
\hline Udder cleft & 19 & 33 & 32 & 32 & 33 & 20 & 36 & 35 & 34 & 36 \\
\hline Udder depth & 17 & 40 & 40 & 40 & 40 & 17 & 42 & 41 & 41 & 42 \\
\hline
\end{tabular}

${ }^{1}$ Parent average from genetic evaluation in 2004 .

$\omega$ values of 0.5 or 0.7 and $\tau=1$ resulted in the least bias without affecting accuracy. This result indicates that contributions from $\mathbf{A}^{22}+\mathbf{G}^{-1}-\omega \mathbf{A}_{22}^{-1}$ (i.e., $\mathbf{A}^{22}-\omega \mathbf{A}_{22}^{-1}$ or $\mathbf{G}^{-1}$ ) are too small to estimate unbiased GEBV for young genotyped bulls. To reduce the inflation of GEBV for bulls with only genomic information, $\mathbf{A}^{22}-\omega \mathbf{A}_{22}^{-1}$ or $\mathbf{G}^{-1}$ can be increased. Table 4 shows that all $\delta$ values with $\omega=0.7$ for ST and MT models were higher and closer to 1.0 than those with $\omega=1.0$. By using $\omega=0.7$ for $\mathbf{A}_{22}^{-1}, \mathbf{H}^{-1}$ for genotyped animals, $\mathbf{A}^{22}+\mathbf{G}^{-1}-\omega \mathbf{A}_{22}^{-1}$, becomes larger than that with no weight $(\omega=1.0)$; therefore, absolute values of GEBV for genotyped will be smaller, resulting in a higher $\delta$ or less bias in GEBV. With $\omega=0.7, \mathrm{R}^{2}$ were the same for both ST and MT models, whereas the $\delta$ values were 0.11 to 0.26 higher for the ST models and 0.12 to 0.23 higher for the MT model with scaled G. In a study involving final scores of US Holsteins (Misztal et al.,

Table 3. Regression $(\delta \times 100)$ of daughter deviations in 2009 on genomic evaluation in 2004 using single-trait and multiple-trait models with equal, base, current, and scaled current allele frequencies for genotyped bulls with at least 50 daughters

\begin{tabular}{|c|c|c|c|c|c|c|c|c|c|c|}
\hline \multirow[b]{2}{*}{ Trait } & \multicolumn{5}{|c|}{ Single-trait model } & \multicolumn{5}{|c|}{ Multiple-trait model } \\
\hline & $\mathrm{PA}^{1}$ & Equal & Base & Current & $\begin{array}{l}\text { Scaled } \\
\text { current }\end{array}$ & $\mathrm{PA}$ & Equal & Base & Current & $\begin{array}{c}\text { Scaled } \\
\text { current }\end{array}$ \\
\hline Stature & 86 & 86 & 83 & 82 & 83 & 87 & 86 & 83 & 84 & 84 \\
\hline Strength & 78 & 78 & 75 & 74 & 75 & 80 & 78 & 76 & 75 & 75 \\
\hline Body depth & 78 & 77 & 75 & 73 & 76 & 78 & 78 & 75 & 74 & 75 \\
\hline Dairy form & 78 & 83 & 79 & 76 & 80 & 78 & 86 & 82 & 78 & 83 \\
\hline Rump angle & 86 & 94 & 89 & 90 & 91 & 85 & 93 & 91 & 90 & 90 \\
\hline Thurl width & 88 & 85 & 82 & 82 & 83 & 87 & 86 & 83 & 81 & 83 \\
\hline Rear legs side view & 82 & 77 & 72 & 72 & 73 & 81 & 79 & 76 & 76 & 76 \\
\hline Foot angle & 75 & 73 & 68 & 68 & 69 & 75 & 72 & 69 & 70 & 69 \\
\hline Fore udder attachment & 70 & 75 & 71 & 70 & 72 & 74 & 78 & 74 & 72 & 75 \\
\hline Rear udder height & 71 & 74 & 67 & 68 & 71 & 69 & 74 & 70 & 66 & 71 \\
\hline Rear udder width & 67 & 71 & 65 & 64 & 68 & 66 & 71 & 67 & 63 & 68 \\
\hline Udder cleft & 84 & 85 & 79 & 80 & 82 & 83 & 87 & 82 & 79 & 84 \\
\hline Udder depth & 81 & 84 & 81 & 80 & 81 & 78 & 85 & 82 & 82 & 83 \\
\hline Front teat placement & 76 & 83 & 78 & 78 & 80 & 76 & 84 & 80 & 77 & 81 \\
\hline Teat length & 75 & 82 & 77 & 77 & 78 & 74 & 83 & 79 & 78 & 79 \\
\hline Rear legs rear view & 69 & 71 & 68 & 62 & 67 & 67 & 67 & 63 & 63 & 63 \\
\hline Feet and legs score & 64 & 60 & 56 & 53 & 56 & 63 & 61 & 57 & 56 & 57 \\
\hline Rear teat placement & 84 & 92 & 85 & 80 & 86 & 80 & 89 & 84 & 81 & 85 \\
\hline
\end{tabular}

${ }^{1}$ Parent average from genetic evaluation in 2004 . 
Table 4. Regression $(\delta \times 100)$ of daughter deviations in 2009 on genomic evaluation in 2004 using single-trait and multiple-trait models with equal, base, current, and scaled current allele frequencies for genotyped bulls with at least 50 daughters with weight $\omega=0.7$ for $\mathbf{A}_{22}^{-1}$ for genotyped bulls

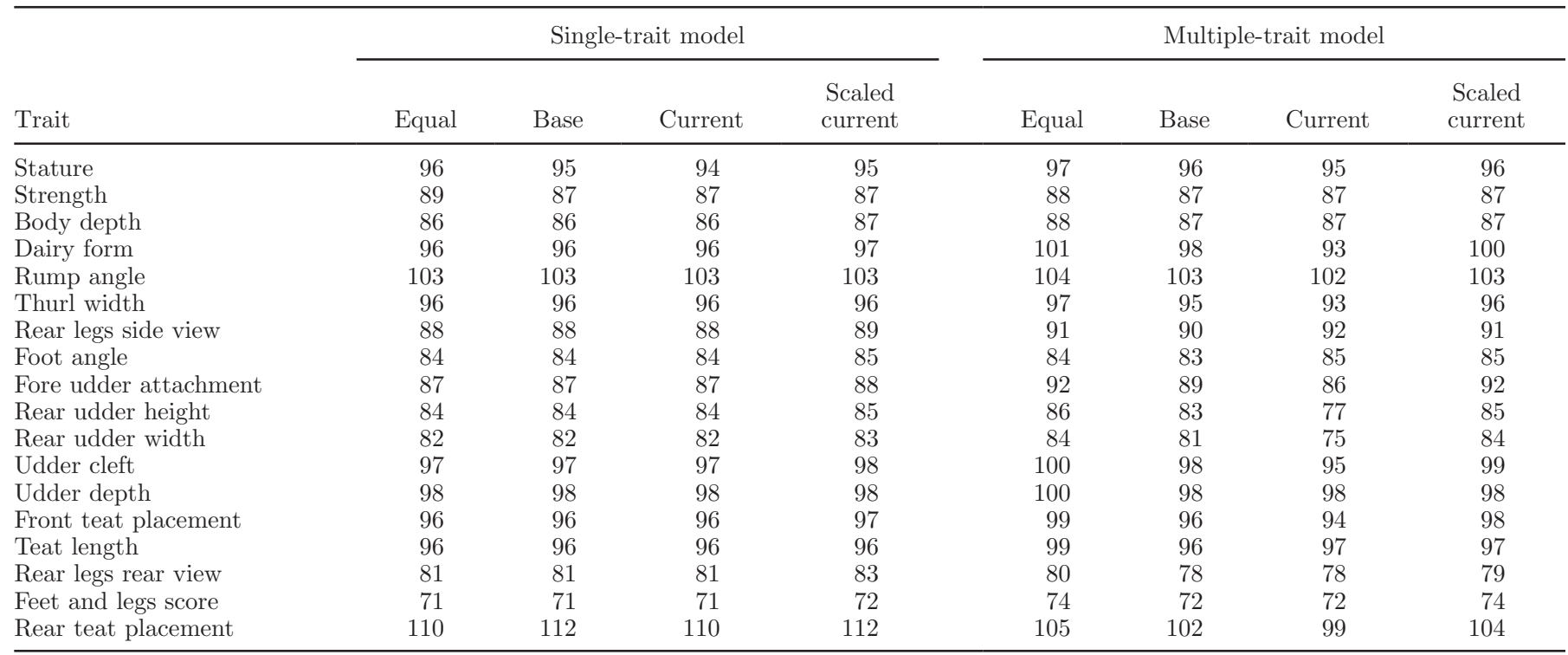

2010), the authors reported that smaller $\omega$ increased $\delta$ but reduced $\mathrm{R}^{2}$ slightly. Assuming that bias of $\pm 15 \%$ is acceptable, 15 out of 18 traits would fall into this category. In addition, the smaller weight for $\mathbf{A}_{22}^{-1}$ did not affect GEBV for old genotyped bulls because $\mathbf{G}^{-1}$ for those bulls was larger than $\mathbf{A}_{22}^{-1}$. A smaller weight (e.g., $\omega=0.5$ ) could produce less-biased GEBV for traits that still have $\delta<1.0$ with small or no reduction in $\mathrm{R}^{2}$ but could underestimate GEBV for traits that have $\delta$ $>1.0$. Therefore, $\omega=0.7$ or 0.5 should be used as a common weight factor for all 18 traits. To reduce bias, the source needs to be determined for any trait. In particular, the elimination of biases in GEBV requires knowledge about their sources with PA. If the sources are unknown, the most effective way to avoid accumulation of biases over multiple cycles of genomic selection may be the continued collection of phenotypes.

\section{CONCLUSIONS}

The multiple-trait single-step approach is feasible for 18 linear type traits in the national genetic evaluation of US Holstein cattle. The use of the multiple-trait model and genomic information leads to an increase in computational costs, whereas the single-trait model does not increase computational time. Accuracy for genomic evaluation increases when switching from singletrait models to multiple-trait models, but the increase depends on traits. Inflation of genomic evaluations for young bulls could be reduced without affecting accuracy by choosing a small weight to account for contributions from the inverse of the numerator relationship matrix for genotyped bulls. Using 0.7 for the weight could be a common factor for all 18 linear type traits; however, further study should be conducted to reduce the bias of genomic evaluations for any traits.

\section{ACKNOWLEDGMENTS}

This study was partially funded by the Holstein Association USA Inc. (Brattleboro, VT) and by AFRI grants 2009-65205-05665 and 2010-65205-20366 from the USDA NIFA Animal Genome Program.

\section{REFERENCES}

Aguilar, I., I. Misztal, D. L. Johnson, A. Legarra, S. Tsuruta, and T. J. Lawlor. 2010. Hot topic: A unified approach to utilize phenotypic, full pedigree, and genomic information for genetic evaluation of Holstein final score. J. Dairy Sci. 93:743-752.

Chen, C. Y., I. Misztal, I. Aguilar, S. Tsuruta, T. H. E. Meuwissen, S. E. Aggrey, T. Wing, and W. M. Muir. 2011. Genome-wide markerassisted selection combining all pedigree phenotypic information and genotypic data in one step: An example using broiler chickens. J. Anim. Sci. 89:23-28.

Christensen, O. F., and M. S. Lund. 2010. Genomic prediction when some animals are not genotyped. Genet. Sel. Evol. 42:2.

Forni, S., I. Aguilar, and I. Misztal. 2011. Different genomic relationship matrices for singlestep analysis using phenotypic, pedigree and genomic information. Genet. Sel. Evol. 43:1.

Gengler, N., P. Mayeres, and M. Szydlowski. 2007. A simple method to approximate gene content in large pedigree populations: Application to the myostatin gene in dual-purpose Belgian Blue cattle. Animal 1:21-28.

Liu, Z., F. Seefried, F. Reinhardt, and R. Reents. 2009. A simple method for correcting the bias caused by genomic pre-selection in conventional genetic evaluation. Pages $184-188$ in Proc. Interbull Annual Mtg., Barcelona, Spain. Inter. Bull Eval. Serv. Bull. No. 40. Dept. Anim. Breeding and Genet., SLU, Uppsala, Sweden. 
Misztal, I., I. Aguilar, A. Legarra, and T. J. Lawlor. 2010. Choice of parameters for single-step genomic evaluation for type. J. Dairy Sci. 93(Suppl. 1):533. (Abstr.)

Misztal, I., A. Legarra, and I. Aguilar. 2009. Computing procedures for genetic evaluation including phenotypic, full pedigree, and genomic information. J. Dairy Sci. 92:4648-4655.

Patry, C., and V. Ducrocq. 2009. Evidence of a bias in genetic evaluation due to genomic selection. Pages 167-171 in Proc. Interbull Annual Mtg., Barcelona, Spain. Inter. Bull Eval. Serv. Bull. No. 40. Dept. Anim. Breeding Genet., SLU, Uppsala, Sweden.

Tsuruta, S., and I. Misztal. 2008. Technical note: Computing options for genetic evaluation with a large number of genetic markers. J. Anim. Sci. 86:1514-1518.
Tsuruta, S., I. Misztal, and T. J. Lawlor. 2005. Changing definition of productive life in US Holstein: Effect on genetic correlations. J. Dairy Sci. 88:1156-1165.

Tsuruta, S., I. Misztal, and I. Stranden. 2001. Use of the preconditioned conjugate gradient algorithm as a generic solver for mixedmodel equations in animal breeding applications. J. Anim. Sci. 79:1166-1172.

VanRaden, P. M. 2008. Efficient methods to compute genomic predictions. J. Dairy Sci. 91:4414-4423.

Vitezica, Z. G., I. Aguilar, and A. Legarra. 2010. One-step vs. multistep methods for genomic prediction in presence of selection. Proc. 9th World Congr. Genet. Appl. Livest. Prod., Leipzig, Germany. CD-ROM Commun. 131. 